\title{
Nigrostriatal dopaminergic depletion increases static orofacial allodynia
}

Wisam Dieb ${ }^{1,2,3^{*}}$, Omar Ouachikh ${ }^{3}$, Sofia Alves ${ }^{3}$, Yves Boucher ${ }^{1,2}$, Franck Durif $^{3,4}$ and Aziz Hafidi ${ }^{3^{*}}$

\begin{abstract}
Background: This study investigated mesencephalic dopamine depletion effects on static mechanical allodynia (SMA) elicited by chronic constriction of the infraorbitary nerve (CCI-loN).

Methods: Dopamine depletion (6-OHDA administration into the medial forebrain bundle) effects on CCl-loNinduced SMA were explored using behavioral (nocifensive behavior score upon non-noxious stimuli using von Frey filament), pharmacological (bromocriptine injections) and immunohistochemical (PKC $y$ and pERK1/2) techniques.

Results: The central dopamine depletion increased significantly the SMA score. Intraperitoneal and intracisternal injections of bromocriptine alleviated the allodynic behavior observed in both CCI-loN and CCl-loN + 6-OHDA animal groups. At the cellular level, dopamine depletion induced a significant increase in PKCY expression in the medullary dorsal horn (MDH) in rat with CCI-IoN + 6-OHDA when compared to sham animals (CCI-loN only). Similarly, after static non-noxious stimuli, the expression of pain marker proteins pERK1/2 within the MDH revealed significantly a higher number of positive cells in CCI-IoN +6-OHDA rats when compared to the CCl-loN group.

Conclusion: This study demonstrates that nigrostriatal dopamine depletion exacerbates the neuropathic pain resulting from $\mathrm{CCl}-\mathrm{loN}$. This effect is probably due to an action through descending pain inhibitory systems which increased pain sensitization at the MDH level. It demonstrates also an analgesic effect elicited by D2R activation at the segmental level.
\end{abstract}

Keywords: Parkinson disease, Dopamine, Pain, Substantia nigra, Chronic constriction injury, Neuropathic pain, Infraorbitary nerve

\section{Background}

Painful traumatic trigeminal neuropathy (PTTN) following peripheral nerve trauma is a disabling condition clinically characterized by spontaneous and evoked pain mainly experienced as burning and/or shooting pain [1]. It results from dysfunctions of the somatosensory system [2] and remains a therapeutic challenge since the current treatment options are unsatisfactory [3]. The physiopathogeny of PTTN points to both peripheral mechanisms involving neuro-glio-immuno vascular alterations mediated by chemokines/cytokines release and central mechanisms involving both alterations of ascending pathways and descending controls [4-7].

\footnotetext{
* Correspondence: wisamdieb@yahoo.com; azhafidi@univ-bpclermont.f 'UFR Odontologie, Université Paris Diderot, Paris, France

${ }^{3}$ Clermont Université, Université d'Auvergne, EA7280, Clermont-Ferrand, France

Full list of author information is available at the end of the article
}

Dopamine has been proposed as playing a key role in chronic orofacial pain [8]. Nigrostriatal dopamine depletion is associated with increased pain sensitivity and is implicated in pain in different pathologies such as Parkinson's disease, restless leg syndrome, fibromyalgia, burning mouth syndrome and atypical facial pain [9-13]. Conversely, striatal administration of dopamine 2 receptor (D2R) agonists has an anti-nociceptive effect mediated by the rostroventromedial medulla (RVM) [14]. Similar results showed that striatal inhibition of nociceptive responses evoked in the trigeminal system [15-17] and chronic oro-facial pain conditions in humans were associated with the alteration of the nigrostriatal dopaminergic system $[9,10]$. Recent reports showed that bilateral or unilateral nigrostriatal dopaminergic lesions induce dynamic and static mechanical allodynia in the oro-facial region $[16,18]$. Since the effects of dopamine depletion on the development of PTTN have
照 Springer

(c) 2016 Dieb et al. Open Access This article is distributed under the terms of the Creative Commons Attribution 4.0 International License (http://creativecommons.org/licenses/by/4.0/), which permits unrestricted use, distribution, and reproduction in any medium, provided you give appropriate credit to the original author(s) and the source, provide a link to the Creative Commons license, and indicate if changes were made. 
not been explored, the present study aimed at investigating the effects of nigrostriatal lesions in animals with a peripheral nerve injury: chronic constriction injury of the Infra Orbitary Nerve (chronic constriction of the infraorbitary nerve: CCI-IoN) model [19]. This model has the advantage of using the trigeminal nerve for pain related studies [20]. Bilateral nigrostriatal chemical lesions were performed by stereotaxic injection of the 6-OHDA toxin into medial forebrain bundle (MFB) in rats with CCI-IoN. In these animals, pain behavior (nocifensive, mechanical allodynia), expression of $\mathrm{PKC} Y$ [(protein involved in pain chronicity) [21, 22]] and pERK1/2 (proteins expressed upon noxious stimuli in the spinal/medullary dorsal horn [23, 24] were assessed with or without dopamine receptor (DR) agonists [bromocriptine (D2R), SKF81297 (D1R)] treatments.

\section{Methods}

\section{Animals}

Adult male Sprague-Dawley rats $(N=112,275-325$ g) from Charles River (L'Arbresle, France) were maintained in a controlled environment (lights on 07:00-19:00, $22^{\circ} \mathrm{C}$ ) with ad libitum access to food and water. The experiments followed the ethical guidelines of the International Association for the Study of Pain, the European Community Council directive of 24 November 1986 (86/609/EEC) and the Animal Ethics Committee of the University of Auvergne.

\section{6-OHDA lesion}

After anesthesia (Ketamine $60 \mathrm{mg} / \mathrm{kg}$, xylazine, $10 \mathrm{mg} / \mathrm{kg}$ ), rats were placed in a stereotaxic frame (David Kopf Instrument, CA, USA) and the MFB were injected bilaterally with 6-OHDA $(0.5 \mu \mathrm{L} / \mathrm{min})$ dissolved in a vehicle solution (0.02\% ascorbate saline) at a concentration of $3 \mu \mathrm{g} / \mu \mathrm{L}$ (Sigma-Aldrich, France) in two deposits (2.25 and $2.85 \mu \mathrm{g}$, respectively) at the following coordinates: anterior (A) -4.0 ; lateral $(\mathrm{L}) \pm 0.8$; ventral $(\mathrm{V})-8.0$; tooth bar at +3.4 and $\mathrm{A}$ $-4.4 ; \mathrm{L} \pm 1.2 ; \mathrm{V}-7.8$; tooth bar at -2.4 [25]. To preserve adrenergic neurons from 6-OHDA toxicity, animals received desipramine (25 mg/kg, i.p., Sigma-Aldrich, France) $30 \mathrm{~min}$ prior to the toxin injection; sham-lesioned rats received only the vehicle at the same coordinates.

\section{$\mathrm{CCl}$-loN surgery}

CCI-IoN was performed following an established surgical procedure $[5,19]$. Briefly, animals were anesthetized using chloral hydrate $(400 \mathrm{mg} / \mathrm{kg}$ i.p.) and an incision of approximately $1 \mathrm{~cm}$ long was made along the gingivobuccal margin, begun just proximal to the first upper molar. About $0.5 \mathrm{~cm}$ of the IoN was freed of adhering tissue and two ligatures ( $4-0$ chromic guts) separated by a 1-2 mm interval were tied loosely around it using 4-0 chromic gut. The sham operation was identical except that the nerve was not ligated.

\section{Behavioral testing and analysis}

The rats were adapted to the observation field $(24 \times 35 \times$ $18 \mathrm{~cm}$ ) and for $30 \mathrm{~min}$ each day for 9 days prior to the beginning of behavioral testing. During this period, the experimenter reached into the cage to apply von Frey $(2 \mathrm{~g})$ stimulus on the animals' faces. For each behavioral testing, the rats were placed in the observation field for a $30 \mathrm{~min}$ period. Stimulation was carried out when the rat was in a sniffing/no locomotion state: with four paws placed on the ground, neither moving nor freezing. The stimulus was applied every $3 \mathrm{~min}$ onto the vibrissal pad (IoN territory). Each series of stimulation consisted of 5 von Frey filament $(2 \mathrm{~g})$ applications every $5 \mathrm{~s}$ alternating on each side of the face. This stimulus is non-noxious.

Behavioral responses were quantified by a blindexperimenter according to the method of [19]: (1) detection, the rats turn heads toward stimulus; (2) withdrawal reaction (the rats turn head away); (3) escape/attack, the rats avoid further contact with the stimulus, or attack the filament; (4) asymmetric grooming, the rats display an uninterrupted series of at least three wash strokes directed at the stimulated area. An absence of response corresponded to a zero score. A mean score value was then calculated for each series of stimulations. All the rats were subjected to 13 sessions of behavioral testing at different time points: before surgery (day 1 ) and after surgery, on weeks $1,2,3,4,5,6,7,8,9,10,11$ and 12 .

\section{Immunohistochemistry}

A day after behavioral experiments, rats were deeply anesthesized with urethane $(1.5 \mathrm{~g} / \mathrm{kg}$ i.p), the vibrissal pads were ipsilaterally stimulated for $2 \mathrm{~min}$ by a von Frey filament $2 \mathrm{~g}$ (60 stimuli delivered, $0.5 \mathrm{~Hz}$ ), and three minutes later, the rats were perfused transcardially with warm $\left(37^{\circ} \mathrm{C}\right.$ ) heparinized saline (25 IU heparin/ml) followed by cold $\left(10{ }^{\circ} \mathrm{C}\right)$ phosphate-buffered solution $(0.1 \mathrm{M}, \mathrm{pH}$ 7.6) containing $4 \%$ paraformaldehyde and $0.03 \%$ picric acid. The brains were placed in $30 \%$ sucrose and $0.05 \%$ sodium azide solution overnight at $4{ }^{\circ} \mathrm{C}$. Brainstem coronal sections $(30 \mu \mathrm{m})$ were cut on a freezing microtome and collected in $0.05 \mathrm{M}$ Tris-buffered saline (TBS).

Free-floating sections were placed in $1 \%$ normal goat serum for $1 \mathrm{~h}$ before overnight incubation at room temperature in primary antibody solutions (mouse antipERK1/2 [1:1000, Cell Signaling Technologies], and rabbit anti-PKCY [1:4000, Sigma-Aldrich and Santa Cruz]. The corresponding secondary antibodies (1:400 for goat antimouse Cy3, 1:200 for goat anti-rabbit Cy2) were incubated at room temperature for $3 \mathrm{~h}$. All antibodies were diluted in TBS containing $0.25 \%$ bovine serum albumin and $0.3 \%$ Triton $\mathrm{X}-100$. The sections were finally rinsed in TBS, mounted onto gelatin-coated slides, dehydrated in alcohol, cleared in xylene, and cover-slipped with distyrene-plasticizer-xylene. The specificity of the 
immunostaining was assessed by omitting primary antibodies, which resulted in the absence of signal.

Immunostaining was analyzed using as motorized Zeiss Axioplan 2 microscope equipped with a Hamamatsu C4742-95 digital camera driven by MetaMorph ${ }^{\circ}$.4 software. In each rat, image acquisition and fluorescent signal quantification were performed from 7 different sections, each taken at a given rostrocaudal plane within the $\mathrm{MDH}$ (from 0 to $-2160 \mu \mathrm{m}$ at $360 \mu \mathrm{m}$ intervals). Brainstem sections were categorized according to their approximate rostrocaudal location from the MDH subnucleus interpolaris junction. pERK1/2 positive cells were counted and data were expressed as the sum of the total number of labeled cells counted from all sections analyzed in each animal [16]. $\mathrm{PKC} Y$ staining was quantified as previously reported [16]. Briefly, PKC $\gamma$ staining was quantified within lamina IIi and the number of positive cells was counted in lamina III. Tyrosine hydroxylase $(\mathrm{TH})$ immunolabelling was performed (anti-TH primary antibody; Millipore, France) as described above. The quantification procedure of the 6-OHDA lesion impact on the SNc was reported previously [25].

\section{Drugs and administration}

Two weeks after the 6-OHDA injection, the animals were briefly $(<3 \mathrm{~min})$ anesthetized with $2 \%$ halothane using a mask and received for intracisternal administration bromocriptine $(7 \mu \mathrm{g} / \mathrm{kg}$ dissolved in $5 \mu \mathrm{l}$ vehicle; Sigma-Aldrich, France) or the vehicle alone ( $5 \mu \mathrm{l}$ of $0.9 \%$ saline) according to our previous results [25]. For i.p. injection we used bromocriptine $(1 \mathrm{mg} / \mathrm{kg})$ and SKF81297 $(3 \mathrm{mg} / \mathrm{kg}$ dissolved in 0.9 \% saline; Sigma-Aldrich, France) concentrations [25]. Following a recovery period $(<2 \mathrm{~min})$, the rats were placed in the observation field for 40-min period-test by a blindexperimenter.

\section{Statistical analysis}

The results are expressed as mean \pm SD. Statistical analysis was performed using Student's t-test, or a one-way analysis of variance (ANOVA) followed by a post hoc Student Newman-Keuls test or a one-way Repeated Measures (RM) ANOVA followed by a post hoc Student-NewmanKeuls test. The level of significance was set at $\mathrm{P}<0.05$.

\section{Results}

Dopamine depletion in the substantia nigra

As shown in our previous study [25], 6-OHDA injections resulted in a considerable decrease of $\mathrm{TH}$ staining in the SNc of CCI-IoN + 6-OHDA when compared to CCI-IoN + saline animals (Additional file 1: Figure S1A and B). Cell count revealed a significant $\left(p<0.001,{ }^{* * * *}\right)$ decrease in $\mathrm{TH}$ positive cells (70\% neuronal loss) in $\mathrm{SNc}$ of CCI-IoN + 6-OHDA rats (Additional file 1: Figure S1C). The impact of unilateral or bilateral depletion mesencephalic midbrain depletion on SMA has been studied previously [18].

\section{Dopamine depletion increases static mechanical allodynia (SMA) resulting from CCI-IoN}

The time course of SMA appearance (Fig. 1), resulting from the CCI-IoN, was similar to our previous report [16].

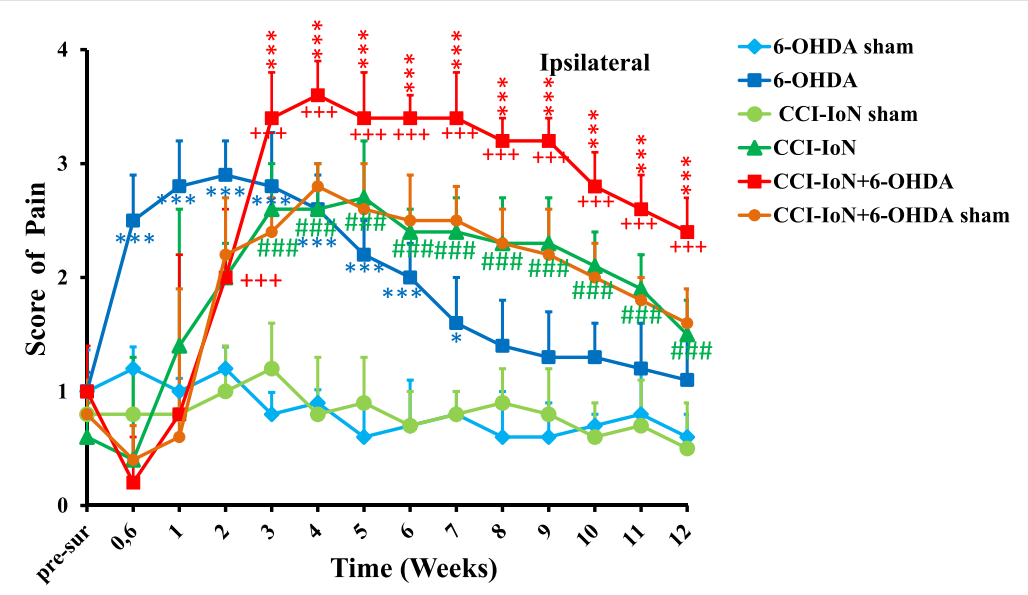

Fig. 1 Time course of static mechanical allodynia (SMA) in the ipsilateral side to the injury (CCI-loN) of 6 animal groups (each $n=8$ ): CCl-loN and its sham; 6-OHDA and its sham; and 6-OHDA + CCI-ION and its sham. CCI-loN animals showed a significant increase in the nocifensive score at post-surgery week 2 when compared to CCl-IoN-sham. The SMA score reached its maximum around the 4 th week post-surgery and decreased slowly through the post-surgery observation period until week 12. SMA occurred rapidly within 3-4 days after 6-OHDA injection. This related SMA stayed significant when compared to sham for 7 weeks after the lesion. The highest nocifensive score was observed in the 6-OHDA+CCI-loN group in comparison to all other groups. Despite the slight decrease observed along the 12 weeks post-surgery, the SMA score in this group remained significantly increased compared to all other groups. $* * *$ Comparison between CCI-ION +6-OHDA and CCI-ION and 6-OHDA. \# Comparison between CCl-loN and CCl-loN sham. + Comparison between CCI-loN +6-OHDA and CCI-loN +6-OHDA sham. * Comparison between 6-OHDA and 6-OHDA sham. The bars represent standard deviation. ${ }^{*} P<0.05 ;{ }^{* *} P<0.01 ;{ }^{* * *} p<0,001$ 
Briefly, 1 week post-surgery, significant SMA score (CCIIoN) was obtained when compared to sham and reached its highest score $\left(p<0.001,{ }^{* * *}\right) 3$ weeks post-surgery. This SMA was significant during 12 weeks post-injury.

The time course of 6-OHDA induced SMA appearance was similar to our previous report [7]. The SMA appeared relatively earlier after 6-OHDA injection than after CCI-IoN (Fig. 1) and stayed significant in comparison to 6-OHDA-sham during the first 6 weeks after the 6-OHDA lesion.

The CCI-IoN + 6-OHDA rats showed the highest significant $\left(p<0.001,{ }^{* * * *}\right)$ SMA score within the ipsilateral side (Fig. 1) to the CCI-IoN injury in comparison to all other animal groups. This score was highly significant along the experiment duration. Similar results were obtained in the contralateral side, although with lower scores (data not shown).

\section{Bromocriptine administration decreases the SMA}

Intraperitoneal administration of bromocriptine induced a significant, dose dependent $(0.1 \mathrm{mg}$ and $1 \mathrm{mg} / \mathrm{Kg})$ decrease in pain scores in CCI-IoN group when compared to sham (Fig. 2a) and its effect lasted for 6 h. The highest dose induced the highest score decrease, $(P<0.01, * *)$. As a positive control SKF8129 (DR1 agonist) was used. Its intraperitoneal administration induced a non-significant increase in the SMA score when compared to sham (saline-injected).

Intracisternal administration of bromocriptine (Fig. 2b) decreased significantly the SMA score when compared

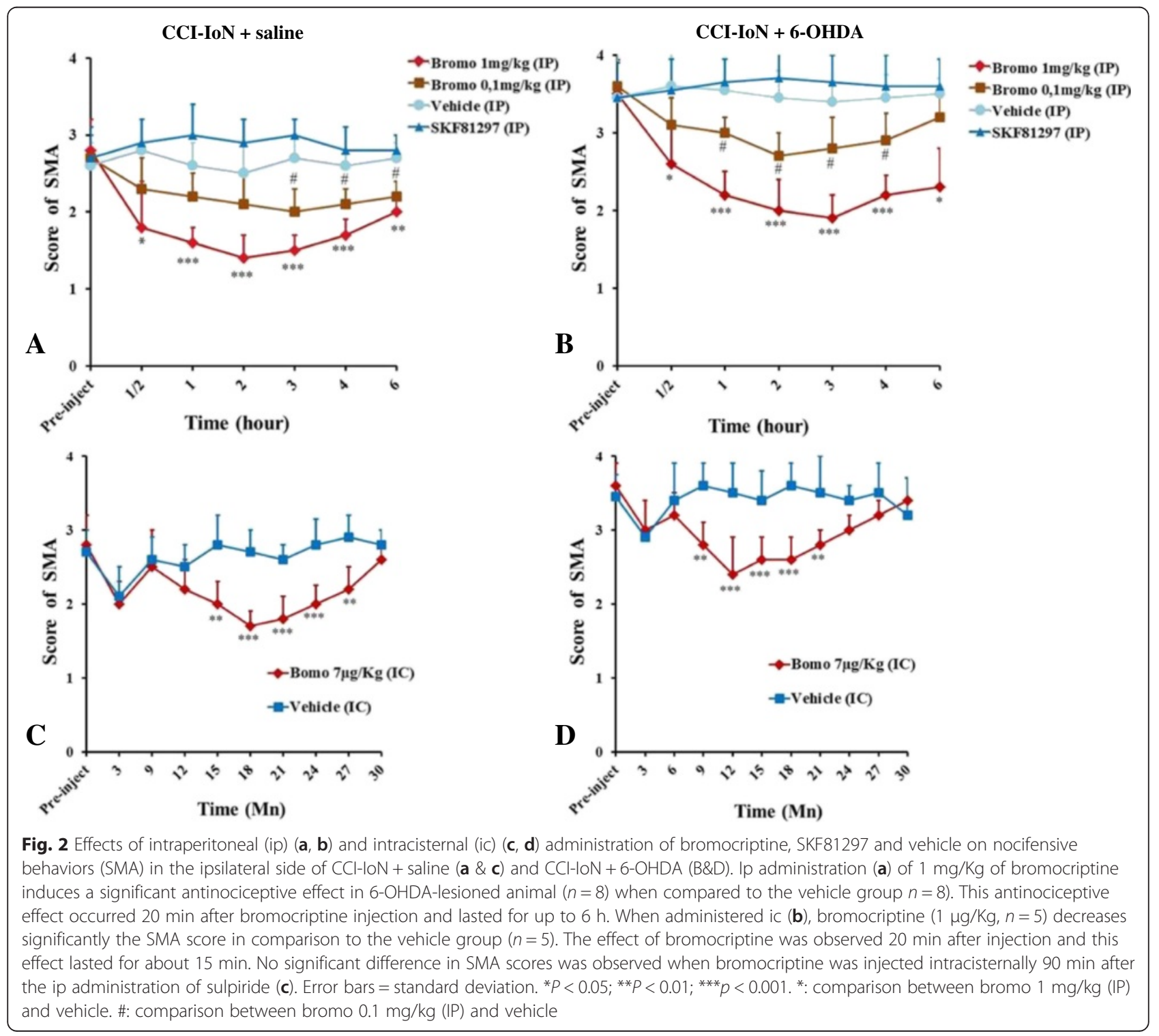


to sham (saline-injected). Bromocriptine effect lasted for $20 \mathrm{~min}$.

Intraperitoneal administration of bromocriptine (Fig. 2c) induced a significant dose dependent decrease in SMA score in CCI-IoN + 6-OHDA lesioned group compared to that of sham. Its effect lasted for 6 h. SKF81297 administration increased the allodynic score, although this score was not significant when compared to sham. Intracisternal administration of Bromocriptine (Fig. 2d) decreased significantly the SMA score compared to that of sham (saline-injected rats) and its effect lasted for $30 \mathrm{~min}$.

\section{PKCY expression in the medullary dorsal horn}

The general pattern of $\mathrm{PKC} \gamma$ staining was similar in both CCI-IoN (Fig. 3a) and CCI-IoN +6-OHDA (Fig. 3b) groups. PKCY staining (Fig. 3c) was highly observed within lamina IIi and in scattered cells within lamina III $[16,22]$. $\mathrm{PKC} \gamma$ staining intensity quantification in lamina IIi (Fig. 3d) was significantly higher in both ipsilateral $\left(P>.001,{ }^{* * *}\right)$ and contralateral $\left(P>.05,{ }^{*}\right) \mathrm{MDH}$ sides of CCI-IoN + 6-OHDA when compared to $\mathrm{CCI}-\mathrm{IoN}$ rats. There was no significant $\mathrm{PKC} \gamma$ staining difference between ipsi- and contralateral sides in lamina IIi. Cell count of $\mathrm{PKC} \gamma$ positive cells in lamina III (Fig. 3e) was significantly increased in CCI-IoN + 6-
OHDA when compared to CCI-IoN groups. There was no significant cell count difference between ipsilateral and contralateral sides.

\section{Increased pERK $1 / 2$ expression in the MDH by 6-OHDA}

pERK $1 / 2$ staining was observed in cells located principally within superficial lamina II and I of the MDH (Fig. 4a-c). At higher magnification the pERK1/2 labeling was observed in cells located mostly in laminae I and IIo (Fig. 4c). Cell count of pERK1/2 positive cells was significantly $(P<.01, * *)$ higher in CCI-IoN + 6-OHDA (Fig 4d) when compared to CCI-IoN injured rats in the ipsilateral side to the injury (stimulated side). There was no significant difference in the number of pERK $1 / 2$ cells between CCI-IoN and CCI-IoN + 6-OHDA in the contralateral side. However there was a significant $\left(P>.01,{ }^{* *}\right)$ cell count difference between ipsi-and contralateral sides in both groups.

\section{PKC $Y$ and pERK $1 / 2$ are distinct cell subtypes}

pERK1/2 (Fig. 5a) and PKCY (Fig. 5b) double labeling revealed no co-localization of these markers in both CCI-IoN and CCI-IoN + 6-OHDA MDH (Fig. 5c).

A summary illustration of the present results is presented in the following table (+low, +++high).
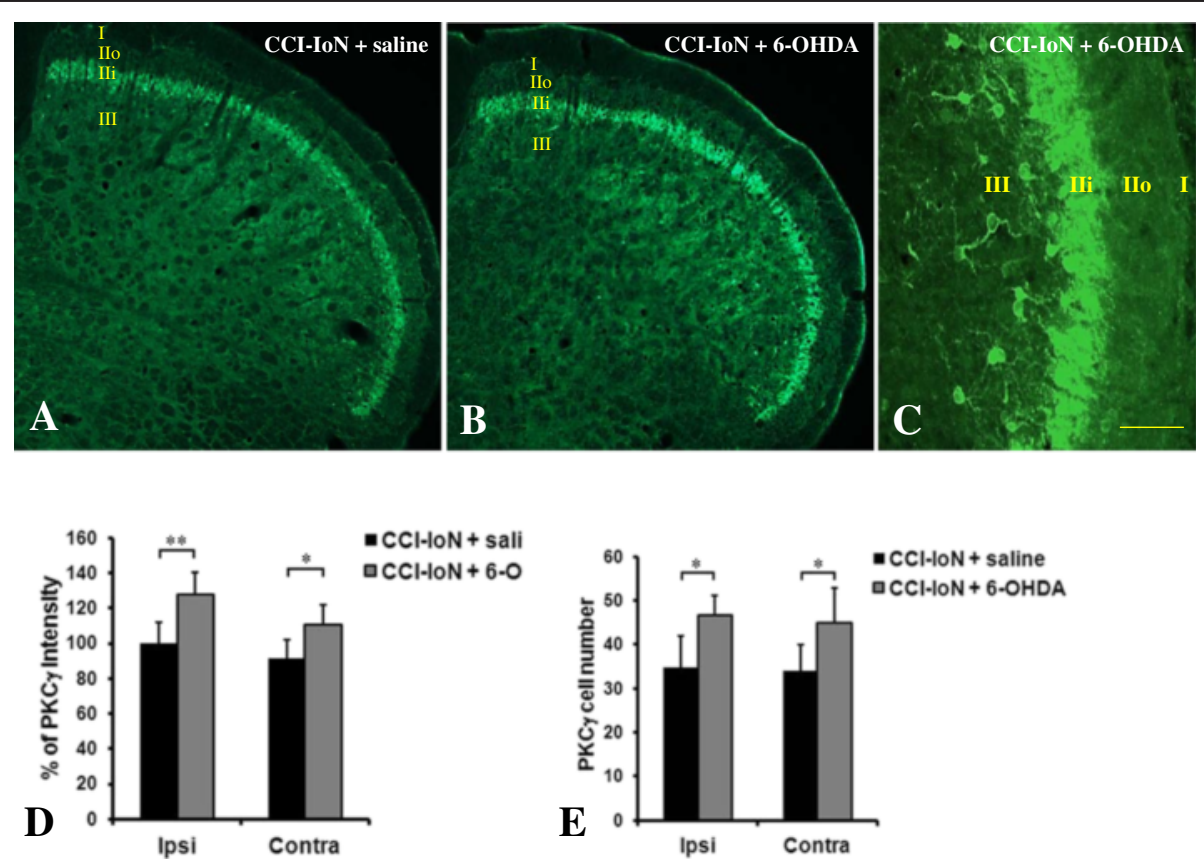

Fig. 3 Expression of PKCy in saline-CCI-ION (a) and 6-OHDA-CCI-loN (b) animals. Intense PKCy labeling was observed within the MDH in lamina Ili cells and in scattered cells of lamina III in both animal groups, but the highest staining signal was observed in the 6-OHDA-group (b). c A high magnification showing the intense label within lamina Ili and in cells within lamina III. PKCY staining intensity analysis (d) showed a significant increase in 6-OHDA injected animals within lamina Ili in both ipsi $(P<0.01)$ and contralateral $(P<0.05)$ sides of the MDH. PKCY positive cell count within lamina III $(\mathbf{e})$ showed a significant increase $(P<0.05)$ in cell number for $6-\mathrm{OHDA}-\mathrm{CCl}-\mathrm{loN}$ compared to saline-CCI-loN animals. Scale bar represents $100 \mu \mathrm{m}$ in $\mathrm{A}-\mathrm{B}$ and $30 \mu \mathrm{m}$ in C 


\begin{tabular}{lccc}
\hline & CCI-ION & 6-OHDA & CCl-loN +6-OHDA \\
\hline SMA & ++ & + & +++ \\
PKCY & ++ & + & +++ \\
pERK & ++ & + & +++ \\
\hline
\end{tabular}

\section{Discussion}

The main results of this study are: 1) Mesencephalic dopamine depletion augmented significantly the pain caused by CCI-IoN. 2) Bromocriptine administrations (intraperitoneal and intracisternal) attenuated SMA in both CCI-IoN and CCI-IoN + 6-OHDA animals. 3) Central dopamine depletion increased significantly $\mathrm{PKC} \gamma$ and pERK $1 / 2$ expressions in the MDH of CCI-IoN + 6-OHDA when compared to CCI-IoN group.

This study shows a synergistic or additional effect of central dopamine depletion and CCI-IoN on SMA in orofacial territories. The dopamine depletion increased the SMA caused by CCI-IoN. This is in agreement with our previous studies demonstrating the induction of dynamic and static $[16,18]$ mechanical allodynia in the trigeminal system upon midbrain dopamine depletion. Bromocriptine attenuated CCI-IoN-related SMA in a dose dependent manner. These results highlights an $\mathrm{MDH}$ local action of bromocriptine by the activation of D2R since SKF81297 (D1R agonist) had no significant effect on pain behavior.
These data are in accordance with previous results showing a direct inhibition of superficial spinal dorsal horn neurons by activation of D2R [26, 27].

At the molecular level, central depletion of dopamine increased synergistically the pre-existing expressions of $\mathrm{PKC} \gamma$ and pERK1/2 within MDH. PKC $\gamma$ is known to be a key molecule for the onset of pain chronicity [21]. Its expression increases after CCI-IoN confirming previous studies [22, 24, 28]. Our result suggests that the lesion of the dopaminergic nigrostriatal system increased the SMA by acting on $\mathrm{PKC} \gamma$ cells through descending pain inhibitory system. PKCY cells are known to activate a secondary cell subtype within $\mathrm{MDH}$ superficial laminae which expressed pERK1/2 [28] and the specific PKCY inhibition decreased both the number of pERK1/2 cells and the related neuropathic pain behavior [24]. This highlights the essential role of $\mathrm{PKC} \gamma$ cells in inducing allodynia. $\mathrm{PKC} \gamma$ cells constitute a revolving door that induces allodynia through peripheral (CCI-IoN) or central lesions (nigrostriatal system). Moreover, bromocriptine administration has been shown in the same neuropathic model to decrease $\mathrm{PKC} \gamma$ expression levels in the $\mathrm{MDH}$ [16], thus suggesting that dopamine might act directly on $\mathrm{PKC} \gamma$ cells either by direct inhibition or indirectly through D2Rs present at excitatory presynaptic site at the
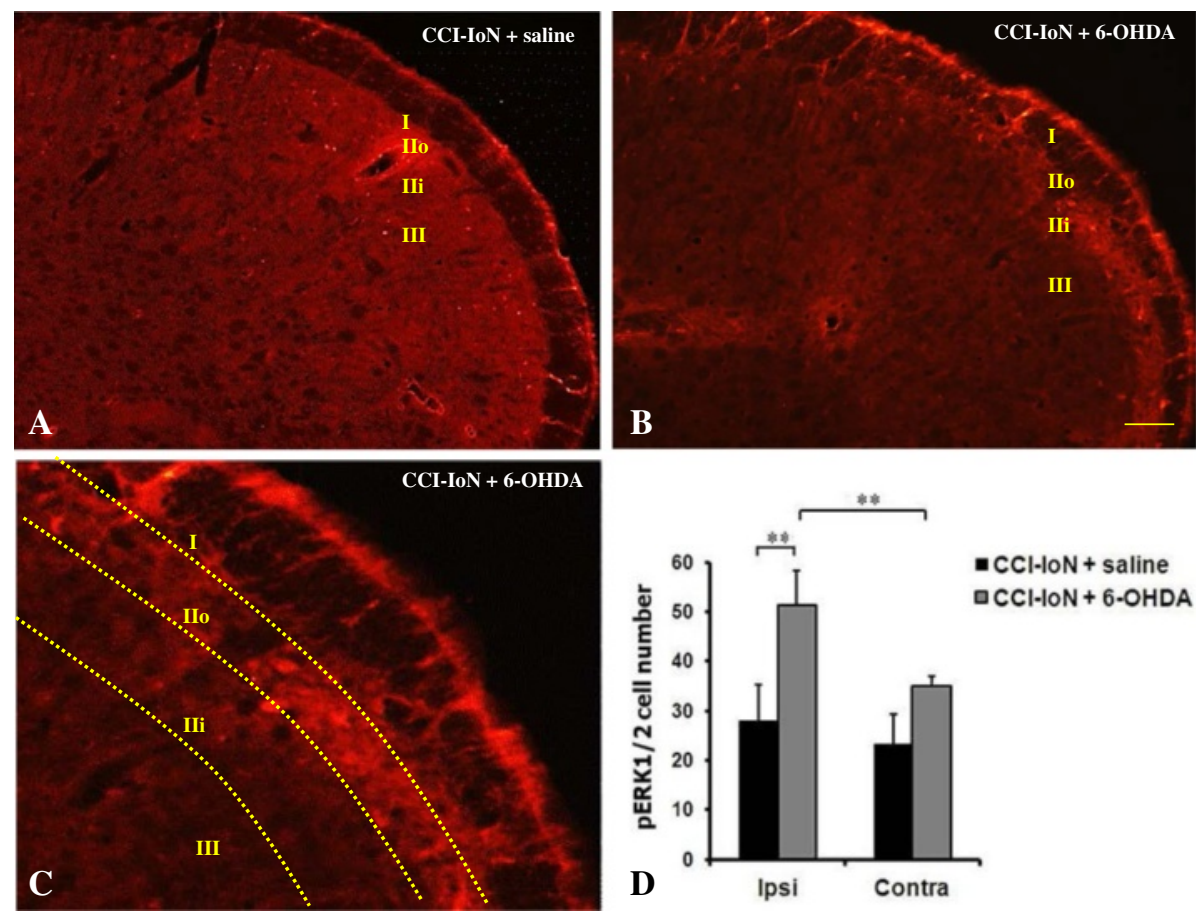

Fig. 4 Expression of pERK1/2 in saline-CCI-ION (a) and 6-OHDA-CCI-loN animals (b-c). pERK1/2 positive cells were observed within superficial laminae of the $\mathrm{MDH}$ in both groups. At high magnification the pERK1/2 positive cells were observed mostly in laminae llo and I as delimited (yellow dots) in the figure (c). Cell count (d) of pERK1/2 positive cells showed a significant increase $\left(P<0.01,{ }^{* *}\right)$ in 6-OHDA vs saline injected animals $(n=5$ each group) in the ipsilateral $\mathrm{MDH}$ and a non-significant increase in the contralateral side. A significant increase in the number of pERK1/2 positive cells was observed in the ipsilateral side of 6-OHDA injected animals compared to the contralateral side. Scale bar represents $100 \mu \mathrm{m}$ in A-B and $40 \mu \mathrm{m}$ in C 

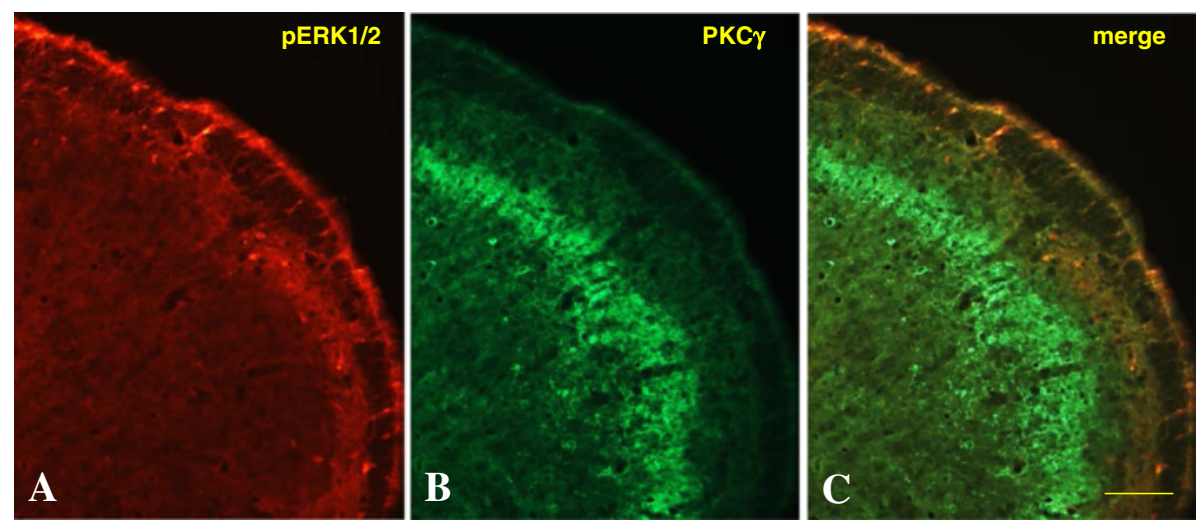

Fig. 5 Double labeling using pERK1/2 (a) and PKCY (b) antibodies in CCI-loN +6-OHDA rats. No co-localization (c) between markers was observed in $\mathrm{MDH}$ cells demonstating distinct cell subtypes for these markers. The bar represents $100 \mu \mathrm{m}$ in A-C

level of $\mathrm{PKC} \gamma$ cells. In support of the latter, D2Rs have been detected post-synaptically on second-order neurons [29].

The implication of basal ganglia, in the processing of noxious somatosensory information is well documented [30]. Activation of the dopaminergic nigrostriatal system leads to a general anti-nociceptive effect [31], while its alteration enhances sensitivity to noxious stimuli [32, 33]. The anti-nociceptive dopamine effect is achieved through D2R receptors $[14,16,31,33]$.

In agreement with these data, the use of bromocriptine in the present study demonstrated also the involvement of D2R receptor in the anti-nociceptive effect resulting from both central dopamine depletion and CCI-IoN.

To date no direct nigral projections to the $\mathrm{MDH}$ is documented. Therefore, the increase of SMA is probably due to the indirect modulation of the pain descending modulatory system through the periaqueductal grey matter (PAG) [34]. The administration of apomorphine (a dopamine receptor agonist) into the PAG promotes anti-nociception [35]. GABA-ergic projections from SNc, substantia nigra reticula, ventral tegmental area and amygdala to the PAG have been described [36, 37]. Dopamine depletion in these structures may decrease GABA transmission at PAG level, thereby increasing the influence of descending facilitatory pain pathways on the $\mathrm{MDH}$ through the RVM. The latter represents the final common pain modulatory system [34]. Stimulation of striatal D2R suppressed nociceptive neuropathic pain through RVM modulation and activation of D2R and 5HT receptors at the dorsal spinal horn [14]. The mesolimbic and meso-cortical dopamine projections can also participate to the increase of pain caused by CCI-IoN since 6-OHDA injection has been demonstrated to induce dopaminergic cell degeneration in the VTA $[16,18,33]$. Alternatively the segmental action of bromocriptine (intracisternal injection) may act via dopaminergic descending pathway which arise from the hypothalamus region A11 [38]. Other brain structures which receive mesencephalic dopamine innervation could also modulate nociception at the MDH level [39].

Our data supported the implication of the mesencephalic dopamine system in the PD, orofacial (burning mouth syndrome, atypical facial pain) and other pain-related pathologies (restless leg, fibromyalgia) $(9.10 .11 .12,13)$. It is worth to note that in $\mathrm{PD}$, patients experimented pain during the Off-period (absence of dopamine replacement therapy) which highlighted the general role for dopamine in pain process. Thus central dopamine may have a general inhibitory action on pain directly by dopaminergic projections to descending pain control or indirectly through dopamine projections to target nuclei and its depletion may causes a general pain increase.

\section{Conclusion}

In conclusion the present study demonstrated a synergic effect of CCI-IoN and central dopamine depletion in neuropathic pain. The nigrostriatal dopamine increased allodynic behavior through D2R at segmental PKC D2R agonists might be used as analgesic mechanism for trigeminal allodynia.

\section{Additional file}

Additional file 1: Figure S1. Tyrosine Hydroxylase $(\mathrm{TH})$ immunostaining
in saline $+\mathrm{CCl}-\mathrm{ION}(\mathrm{A})$ and $6-\mathrm{OHDA}+\mathrm{CCl}-\mathrm{loN}(\mathrm{B})$ animals $(n=8)$ revealed
a drastic decrease in the staining intensity mainly observed in the substantia
nigra pars compacta $(\mathrm{SNC})$. The cell count $(\mathrm{C})$ demonstrated a significant
$\left(p<0.001,{ }^{* *}\right)$ decrease in $\mathrm{TH}$ positive cells in the SNc of $6-\mathrm{OHDA}$
rats when compared to shams. Scale bar $=170 \mu \mathrm{m}$ in A-B. Error bar $=$ standard
deviation in $\mathrm{C}^{* * *} p<0.001 .(\mathrm{DOC} 274 \mathrm{~kb})$

Competing interests

The authors declare that they have no competing interests. 


\section{Authors' contributions}

WD done the experiments, participate to the writting and discussion of the manuscript. $\mathrm{OO}$ done the experiment and participate to the discussion of the manuscript. SA done the experiment. YB particpate to the writting and the discussion of the manuscript. FD particpate to the discussion of the manuscript. AH participate to the elaboration of the project, writting and the discussion of the paper. All authors read and approved the final manuscript.

\section{Acknowledgement}

This work was supported by funding from Auvergne University.

\section{Author details}

'UFR Odontologie, Université Paris Diderot, Paris, France. ${ }^{2}$ Centre de Psychiatrie et Neurosciences, INSERM U894, Paris, France. ${ }^{3}$ Clermont Université, Université d'Auvergne, EA7280, Clermont-Ferrand, France. ${ }^{4} \mathrm{CHU}$ Clermont-Ferrand, Service de Neurologie, 63000 Clermont-Ferrand, France.

\section{Received: 30 September 2015 Accepted: 12 February 2016} Published online: 17 February 2016

\section{References}

1. Benoliel R, Zadik Y, Eliav E, Sharav Y (2012) Peripheral painful traumatic trigeminal neuropathy: clinical features in 91 cases and proposal of novel diagnostic criteria. J Orofac Pain 26:49-58

2. Jensen TS, Baron R, Haanpää M, Kalso E, Loeser JD, Rice ASC, Treede R-D (2011) A new definition of neuropathic pain. Pain 152:2204-2205

3. Haviv Y, Zadik Y, Sharav Y, Benoliel R (2014) Painful traumatic trigeminal neuropathy: an open study on the pharmacotherapeutic response to stepped treatment. J Oral Facial Pain Headache 28:52-60

4. Iwata K, Tsuboi Y, Shima A, Harada T, Ren K, Kanda K, Kitagawa J (2004) Central neuronal changes after nerve injury: neuroplastic influences of injury and aging. J Orofac Pain 18:293-298

5. Dieb W, Hafidi A (2013) Astrocytes are involved in trigeminal dynamic mechanical allodynia: potential role of D-serine. J Dent Res 92:808-813

6. Okubo M, Castro A, Guo W, Zou S, Ren K, Wei F, Keller A, Dubner R (2013) Transition to persistent orofacial pain after nerve injury involves supraspinal serotonin mechanisms. J Neurosci 33:5152-5161

7. Dauvergne C, Molet J, Reaux-Le Goazigo A, Mauborgne A, MélikParsadaniantz S, Boucher Y, Pohl M (2014) Implication of the chemokine CCL2 in trigeminal nociception and traumatic neuropathic orofacial pain. Eur J Pain 18:360-375

8. Wood PB (2008) Role of central dopamine in pain and analgesia. Expert Rev Neurother 8:781-797

9. Hagelberg N, Forssell H, Aalto S, Rinne JO, Scheinin H, Taiminen T, Någren K, Eskola O, Jääskeläinen SK (2003a) Altered dopamine D2 receptor binding in atypical facial pain. Pain; 106: 43-48

10. Hagelberg N, Forssell H, Rinne JO, Scheinin H, Taiminen T, Aalto S, Luutonen S, Någren K, Jääskeläinen SK (2003b) Striatal dopamine D1 and D2 receptors in burning mouth syndrome. Pain. 101: 149-154

11. Cervenka S, Pålhagen SE, Comley RA, Panagiotidis G, Cselényi Z, Matthews JC, Lai RY, Halldin C, Farde L (2006) Support for dopaminergic hypoactivity in restless legs syndrome: a PET study on D2-receptor binding. Brain J Neurol 129:2017-2028

12. Ford B (2010) Pain in Parkinson's disease. Mov Disord 25:S98-S103

13. Valkovic P, Minar M, Singliarova H, Harsany J, Hanakova M, Martinkova J, Benetin J (2015) Pain in Parkinson's Disease: A Cross-Sectional Study of Its Prevalence, Types, and Relationship to Depression and Quality of Life. PLoS ONE 10:e0136541

14. Ansah $\mathrm{OB}$, Leite-Almeida $H$, Wei $H$, Pertovaara $A$ (2007) Striatal dopamine D2 receptors attenuate neuropathic hypersensitivity in the rat. Exp Neurol 205:536-546

15. Belforte JE, Pazo JH (2005) Striatal inhibition of nociceptive responses evoked in trigeminal sensory neurons by tooth pulp stimulation. J Neurophysiol 93:1730-1741

16. Dieb W, Ouachikh O, Durif F, Hafidi A (2014) Lesion of the dopaminergic nigrostriatal pathway induces trigeminal dynamic mechanical allodynia. Brain Behav 4:368-380

17. Barceló AC, Fillipini B, Pazo JH (2010) Study of the neural basis of striatal modulation of the jaw-opening reflex. J Neural Transm 117:171-181
18. Dieb W, Ouachikh O, Durif F, Hafidi A (2015) Nigrostriata dopaminergic depletion produces orofacial static mechanical allodynia. Eur J Pain 29:70-82

19. Vos BP, Strassman AM, Maciewicz RJ (1994) Behavioral evidence of trigeminal neuropathic pain following chronic constriction injury to the rat's infraorbital nerve. J Neurosci 14:2708-2723

20. Kernisant M, Gear RW, Jasmin L, Vit JP, Ohara PT (2008) Chronic constriction injury of the infraorbital nerve in the rat using modified syringe needle. J Neurosci Methods 172:43-47

21. Malmberg AB, Chen C, Tonegawa S, Basbaum Al (1997) Preserved acute pain and reduced neuropathic pain in mice lacking PKCgamma. Science 278:279-283

22. Nakajima A, Tsuboi Y, Suzuki I, Honda K, Shinoda M, Kondo M, Matsuura S, Shibuta K, Yasuda M, Shimizu N, Iwata K (2011) PKCgamma in Vc and C1/C2 is involved in trigeminal neuropathic pain. J Dent Res 90:777-781

23. Ji RR, Baba H, Brenner GJ, Woolf CJ (1999) Nociceptive-specific activation of ERK in spinal neurons contributes to pain hypersensitivity. Nat Neurosci 2:1114-1119

24. Dieb W, Alvarez P, Hafidi A (2015) PKCY-positive neurons gate light tactile inputs to pain pathway through pERK1/2 neuronal network in trigeminal neuropathic pain model. J Oral Facial Pain Headache 29:70-82

25. Ouachikh O, Dieb W, Durif F, Hafidi A (2013) Differential behavioral reinforcement effects of dopamine receptor agonists in the rat with bilateral lesion of the posterior ventral tegmental area. Behav Brain Res 252: 24-31

26. Tamae A, Nakatsuka T, Koga K, Kato G, Furue H, Katafuchi T, Yoshimura M (2005) Direct inhibition of substantia gelatinosa neurones in the rat spinal cord by activation of dopamine D2-like receptors. J Physiol 568:243-253

27. Taniguchi W, Nakatsuka T, Miyazaki N, Yamada H, Takeda D, Fujita T, Kumamoto E, Yoshida M (2011) In vivo patch-clamp analysis of dopaminergic antinociceptive actions on substantia gelatinosa neurons in the spinal cord. Pain 152:95-105

28. Dieb W, Hafidi A (2015) Mechanism of GABA involvement in post-traumatic trigeminal neuropathic pain: activation of neuronal circuitry composed of PKC $y$ interneurons and pERK1/2 expressing neurons. Eur J Pain 19:85-96

29. Bergerot A, Storer RJ, Goadsby PJ (2007) Dopamine inhibits trigeminovascular transmission in the rat. Ann Neurol 61:251-262

30. Chudler EH, Dong WK (1995) The role of the basal ganglia in nociception and pain. Pain 60:3-38

31. Magnusson JE, Fisher K (2000) The involvement of dopamine in nociception: the role of $D(1)$ and $D(2)$ receptors in the dorsolateral striatum. Brain Res 855:260-266

32. Saadé NE, Atweh SF, Bahuth NB, Jabbur SJ (1997) Augmentation of nociceptive reflexes and chronic deafferentation pain by chemical lesions of either dopaminergic terminals or midbrain dopaminergic neurons. Brain Res 751:1-12

33. Takeda R, Ikeda T, Tsuda F, Abe H, Hashiguchi H, Ishida Y, Nishimori T (2005) Unilateral lesions of mesostriatal dopaminergic pathway alters the withdrawal response of the rat hindpaw to mechanical stimulation. Neurosci Res 52:31-36

34. Millan MJ (2002) Descending control of pain. Prog Neurobiol 66:355-474

35. Meyer PJ, Morgan MM, Kozell LB, Ingram SL (2009) Contribution of dopamine receptors to periaqueductal gray-mediated antinociception. Psychopharmacology (Berl) 204:531-540

36. Chieng B, Christie MJ (2010) Somatostatin and nociceptin inhibit neurons in the central nucleus of amygdala that project to the periaqueductal grey. Neuropharmacology 59:425-430

37. Kirouac GJ, Li S, Mabrouk G (2004) GABAergic projection from the ventral tegmental area and substantia nigra to the periaqueductal gray region and the dorsal raphe nucleus. J Comp Neurol 469:170-184

38. Hökfelt T, Phillipson O, Goldstein M (1979) Evidence for a dopaminergic pathway in the rat descending from the A11 cell group to the spinal cord. Acta Physiol Scand 107:393-395

39. Malmierca E, Martin YB, Nuñez A (2012) Inhibitory control of nociceptive responses of trigeminal spinal nucleus cells by somatosensory corticofugal projection in rat. Neuroscience 221:115-124 